\title{
The effect of chronic alcohol intoxication and smoking on the output of salivary immunoglobulin $A$
}

\author{
Napoleon Waszkiewicz ${ }^{1}$, Anna Zalewska ${ }^{2}$, Slawomir Dariusz Szajda ${ }^{3}$, \\ Magdalena Waszkiewicz ${ }^{4}$, Agata Szulc ${ }^{1}$, Alina Kepka ${ }^{5}$, Beata Konarzewska', \\ Alina Minarowska ${ }^{6}$, Beata Zalewska-Szajda ${ }^{7}$, Dorota Wilamowska ${ }^{3}$, \\ Danuta Waszkiel $^{2}$, Jerzy Robert Ladny ${ }^{3}$, Krzysztof Zwierz ${ }^{8}$
}

${ }^{1}$ Department of Psychiatry, Medical University of Białystok, Poland

${ }^{2}$ Department of Paedodontics, Medical University of Białystok, Poland

${ }^{3}$ Department of Emergency Medicine and Disasters, Medical University of Białystok, Poland

${ }^{4}$ Neurology II unit, Regional Hospital in Białystok, Poland

${ }^{5}$ Department of Biochemistry and Experimental Medicine, the Children's Memorial Health Institute, Warsaw, Poland

${ }^{6}$ Department of Anatomy, Medical University of Bialystok, Poland

${ }^{7}$ Department of Imaging Diagnostics, Children's Hospital, Medical University of Bialystok, Poland

${ }^{8}$ Medical College of the Universal Education Society, Łomża, Poland

\begin{abstract}
The effect of chronic alcohol intoxication and smoking on the output of salivary immunoglobulin A (IgA) was studied in 37 volunteers: 17 male smoking patients after chronic alcohol intoxication (AS) and 20 control non-smoking male social drinkers (CNS). The DMFT index (decayed, missing, or filled teeth), gingival index and papilla bleeding index (PBI) were assessed. Concentration of IgA in saliva was determined by ELISA. Salivary flow (SF) and IgA output were significantly decreased in AS compared to CNS. There were no significant correlations between the amount of alcohol/cigarettes as well as the duration of alcohol intoxication/smoking, and SF or IgA output, nor between IgA level and SF. Gingival index was significantly higher in AS than in CNS, and was inversely correlated with IgA salivary level. The worsened periodontal state in smoking alcoholdependent persons may result from diminished IgA protection of the oral tissues due to its decreased output. (Folia Histochemica et Cytobiologica 2012, Vol. 50, No. 4, 605-608)
\end{abstract}

Key words: alcohol, smoking, saliva, gingival index, IgA

\section{Introduction}

As much as $3.8 \%$ of global deaths, $4.6 \%$ of diseases and injuries, and $30 \%$ of health care costs, are attributable to alcohol abuse. About $2 \%$ of the population is dependent on alcohol [1]. About $80 \%$ of alcoholics

Correspondence addresse: N. Waszkiewicz, Department of Psychiatry, Medical University of Bialystok, 16-070 Choroszcz, Poland; tel./fax: +48 8571939 77; e-mail: napoleonwas@yahoo.com; napwas@wp.pl smoke cigarettes [2]. Damage to the oral tissues following alcohol and nicotine intoxication is mostly attributed to acetaldehyde action. However, some effects of alcohol intoxication result also from the direct action of ethanol, reactive oxygen species (ROS) and fatty acid ethyl esters (FAEEs) [2,3]. Although oral mucosa may allow the entry of many pathogens to the system of innate and acquired immunity of oral mucosa as well as saliva, they usually eliminate the majority of pathogens and prevent massive overgrowth of microorganisms. Salivary proteins not only 
participate in the protection of the oral cavity, but also in the protection of the upper gastrointestinal and respiratory tracts [4].

Immunoglobulin A (IgA) is a glycoprotein that participates in the acquired immune response. Salivary secretory IgA represents more than $85 \%$ of the total salivary immunoglobulin content, which is ten times more than IgG and a hundred times more than IgM content. Salivary IgA is produced by the mucosaassociated lymphoid tissue represented in the mouth and throat by tonsils and other organised lymphoid tissue (e.g. Waldeyer's ring), which expand after direct stimulation by antigens [5]. The precursor B cell of IgA migrates from the gut-associated lymphoid tissue to salivary glands, after local antigenic stimulation in the gut [5]. After antigen stimulation, infiltrating plasma cells may produce IgA in salivary glands. IgAs then acquire Ig receptor of epithelial cells to be secreted as a dimer of s-IgA [5, 6]. Salivary IgAs may also partially derive from paracellular transport (from the blood and the intercellular space), which gains importance when the mucous membrane and epithelium are irritated [5]. IgA can neutralise viruses, bacteria and their toxins. By binding bacterial antigens, IgAs aggregate them and inhibit their adhesion to the oral tissues $[4,7]$. By inhibiting the activity of some hydrolases (e.g. hyaluronidase, chondroitinsulfatase, or neuraminidase) produced by bacteria, IgAs prevent the destruction of periodontal tissues [5-7]. There are fluctuations in the IgA level during the day, with the lowest concentration in the morning $[8,9]$. $\operatorname{IgA}$ is very sensitive to external factors such as stress, anxiety, and sleep deprivation. Intensive physical exercise usually reduces IgA levels in the saliva. Eating spicy food increases the level of T cells, immunoglobulin-producing B cells, and subsequent IgAs production $[8,9]$. Some people with salivary $\operatorname{IgA}$ deficiency may suffer from upper respiratory tract infections [9], although there is no clear correlation between serum IgAs and susceptibility for infection. Moreover, the IgA deficiency may be compensated for by increased $\mathrm{IgG}$ and $\mathrm{IgM}$ levels, or by elevated levels of innate immune proteins [9].

The aim of this study was to determine the effect of chronic alcohol consumption and cigarette smoking on the output of salivary immunoglobulin A.

\section{Material and methods}

A total of 37 volunteers participated in the study: 17 male alcohol-dependent smoking patients (AS) admitted to the Detoxification Unit after chronic alcohol intoxication (mean age: 42 years; range: $26-55 ; 100-700$ g/day of alcohol; 10-20 cigarettes/day) and 20 control male social drinkers (CNS; control non-smokers; mean age: 42 years; range: $30-53$ ). The AS group individuals met criteria for alcohol and nicotine dependence according to ICD-10 (the average time of dependence was 15 years for alcohol and 20 years for smoking). The length of the alcohol intoxication ranged from three to 90 days (mean $\sim 30$ ). Saliva was collected on the second day of the abstinence period as described below. The study was approved by the Bioethical Committee of the Medical University of Bialystok. Informed written consent was obtained from all the subjects after explanation of the nature, purpose, and potential risks of the study.

A check-up of the oral cavity was done by one qualified dentist, following the World Health Organisation criteria [4]. The DMFT, GI, and PBI indices of our subjects were: in alcohol-dependent subjects $19.5 \pm 5.7,0.99 \pm 0.76$, and $0.57 \pm 0.27$, respectively, and in controls $18.8 \pm 5.70,0.30 \pm$ \pm 0.47 , and $0.35 \pm 0.48$, respectively. The subjects were instructed to refrain from smoking, food and beverages, except water, for two hours before saliva was collected. All salivary samples ( $3 \mathrm{ml}$ of resting whole saliva) were collected to plastic tubes placed on ice by the spitting method, under standardised conditions [2], between 8:00 and 9:00 am, to minimise the influence of circadian rhythms. The samples were centrifuged at $3,000 \times \mathrm{g}$ for 20 minutes at $4^{\circ} \mathrm{C}$, to remove cells and debris. Salivary flow (SF) was calculated by dividing the volume of saliva by the time of its collection.

Concentrations of $\operatorname{IgA}$ were assessed by the Human $\operatorname{IgA}$ ELISA Quantitation Kit (Bethyl Laboratories, Cat No. E80102, Montgomery, TX, USA), according to the manufacturer's instructions.

Statistical analysis was performed with Statistica 8.0 (Statsoft, Krakow, Poland). The differences between groups were evaluated using a Mann-Whitney U test. Spearman's rank correlation coefficient was used to measure the statistical dependence between two variables. Statistical significance was assumed at $\mathrm{p}<0.05$.

\section{Results and discussion}

Salivary flow (SF) was significantly lower in smoking alcohol-dependent persons $(0.31 \pm 0.31)$ than in the control group $(0.43 \pm 0.10 \mathrm{~mL} / \mathrm{min})(\mathrm{p}=0.007)$ (Figure 1). There was a tendency to decreased concentration of IgA in the saliva of smoking alcohol-dependent persons $(88 \pm 32 \mathrm{mg} / \mathrm{L})$ compared to the control group $(117 \pm 42 \mathrm{mg} / \mathrm{L})(\mathrm{p}=0.070)$. Salivary IgA output was significantly decreased in smoking alcohol-dependent persons ( $14.6 \pm 10 \mathrm{mg} / \mathrm{min})$ compared to the control group $(51.9 \pm 23 \mathrm{mg} / \mathrm{min})(\mathrm{p}=0.0005)$ (Figure 2). Gingival index (GI) was significantly higher in smoking alcohol-dependent persons $(0.99 \pm 0.76)$ than in the control group $(0.30 \pm 0.47)(p=0.003)$. A significant inverse correlation was found between IgA output and GI $\left(\mathrm{r}^{2}=-0.57, \mathrm{p}=0.004\right)$. There were no 


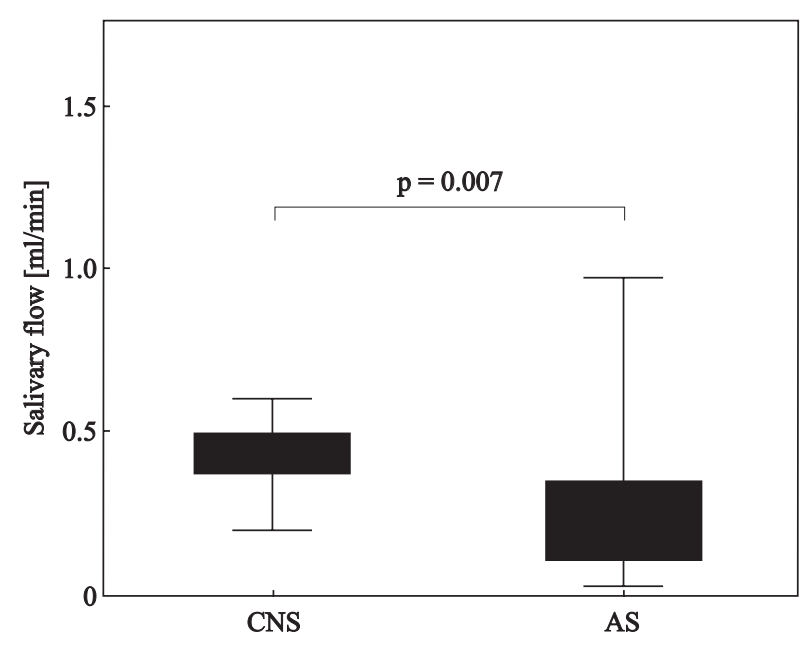

Figure 1. Salivary flow (SF) in control non-smokers (CNS) and smoking alcohol-dependent persons (AS)

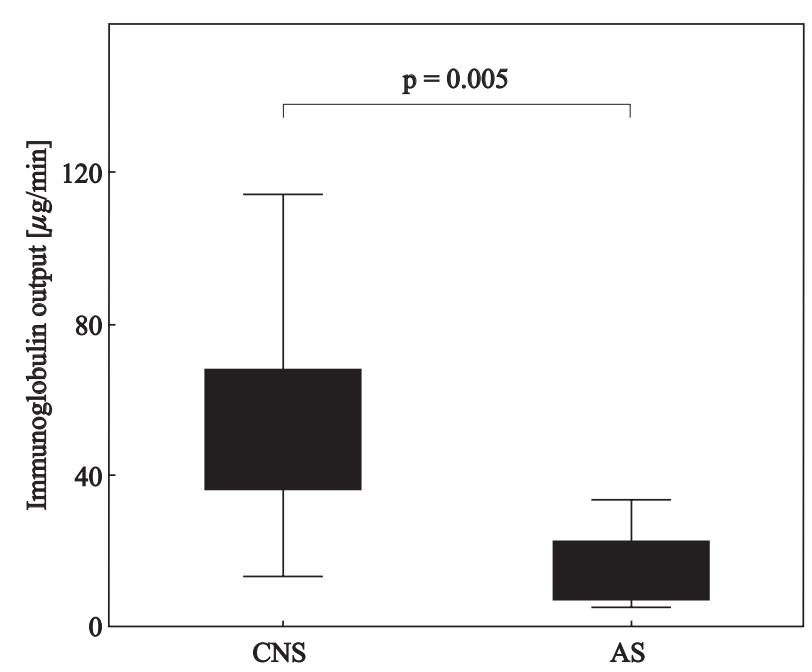

Figure 2. Immunoglobulin A ( $\operatorname{IgA})$ output in control non-smokers (CNS) and smoking alcohol-dependent persons (AS)

significant differences in PBI and DMFT index between smoking alcohol-dependent persons and the control group. No statistically significant correlations were found between the amount and length of alcohol consumption as well as cigarette smoking, and the salivary concentration or IgA output. Also, no significant correlations were found between salivary $\operatorname{IgA}$ output/concentration and salivary flow.

As neither concentration nor output of the IgA showed significant correlation with the amount and duration of alcohol consumption or cigarette smoking, this may indicate an indirect mechanism of specific immunity reduction by alcoholism/smoking in the oral tissues. Significantly higher gingival index (GI) in alcoholics may suggest an inflammatory IgA origin in the saliva, due to worse periodontal condition of addicted persons compared to control subjects. It has been found that chronic alcohol intoxication reduced the number of T, B and NK cells in the thymus, spleen and lymphatic nodes, leading to atrophy of lymphoid organs and reducing the humoral and cellular immune response [10]. Deficiency of lymphocytes in Peyer's patches of gastrointestinal mucosa, in the course of alcohol dependence, and impairment in antigen presentation by affected antigen-presenting cells, resulted in decreased secretion of immunoglobulins [10].

Our findings suggest that increased GI in alcoholdependent patients resulted from the lack of protective effect of $\operatorname{IgA}$ on the periodontium. It is known that IgA secretion is very sensitive to external factors. Stress, anxiety, sleep deprivation, and intensive physical exercise reduce the concentration of $\operatorname{IgA}$ in the saliva $[8$, 9]. Hence the severe stress during alcohol withdrawal syndrome in subjects addicted to alcohol might reduce the output of IgA. A high level of cortisol, accompanying huge abstinence stress, has been shown to correlate with the severity of withdrawal symptoms [11]. Additionally, a negative correlation has been reported between salivary cortisol and IgA levels [9].

A number of epidemiological studies have shown an association between heavy alcohol drinking and poor dental status and oral hygiene [2]. It has also been found that persons addicted to alcohol with bad dental status have a double concentration of acetaldehyde in the saliva compared to those addicted to alcohol but who look after their oral hygiene [2]. In alcohol abusers, reduced salivary flow (SF) may lead to inflammatory state of the oral cavity, periodontal disease, or infection [12].

Non-smokers represent only about $20 \%$ of alcoholics [2]. Smoking is usually associated with poor oral hygiene status and severity of periodontal disease, including periodontitis [2]. Similarly to alcohol, cigarette smoke is a source of oxidative stress, and high concentrations of acetaldehyde in the oral cavity [2]. Smoking has been associated with decreased secretion of salivary proteins, impairment of the mechanisms of both innate and acquired immunity such as phagocytosis, chemotaxis of neutrophils (even in occasional smokers), increased production of peroxides, impaired function and proliferation of B and T lymphocytes, and reduced levels of immunoglobulin $G$ [13]. It has been shown that concomitant alcohol abuse and cigarette smoking resulted in a synergistic increase in the concentration of acetaldehyde in the saliva [2, 14].

The lack of correlations, found in our study, between the amount of alcohol consumed and cigarettes 
number and duration of alcohol intoxication/smoking, with salivary flow and IgA concentration/output, as well as with GI, PBI and DMFT values, suggests indirect damaging tissue effects of drinking and smoking on the immunologic status of oral tissues. On the other hand, it is known that smoking alone increases temporarily resting and stimulated salivary flow, which is due to the irritating effect of tobacco smoke on the mucosa [2]. Therefore, in our study, SF reduction was the result of alcohol intoxication, rather than smoking.

In conclusion, we found that chronic ethanol intoxication in smokers reduced salivary flow and salivary IgA output which was due to ethanol action rather than smoking. Bad periodontal status of smoking alcoholdependent patients could result from the diminished protective effect of salivary $\operatorname{IgA}$.

\section{References}

1. Waszkiewicz N, Szajda SD, Kępka A, Szulc A, Zwierz K. Glycoconjugates in the detection of alcohol abuse. Biochem Soc Trans. 2011;39:365-369.

2. Waszkiewicz N, Chojnowska S, Zalewska A, Zwierz K, Szulc A, Szajda SD. Salivary hexosaminidase in smoking alcoholics with bad periodontal and dental states. Drug Alcohol Depend. (2012), in press.

3. Waszkiewicz N, Szajda SD, Zalewska A et al. Alcohol abuse and glycoconjugate metabolism. Folia Histochem Cytobiol. 2012;50:1-11.
4. Waszkiewicz N, Szajda SD, Jankowska A et al. The effect of acute ethanol intoxication on salivary proteins of innate and adaptive immunity. Alcohol Clin Exp Res. 2008;32:652-656.

5. Marcotte H, Lavoie MC. Oral microbial ecology and the role of salivary immunoglobulin A. Microbiol Mol Biol Rev. 1998;62:71-109.

6. Johansen FE, Pekna M, Norderhaug IN et al. Absence of epithelial immunoglobulin A transport, with increased mucosal leakiness, in polymeric immunoglobulin receptor/secretory component-deficient mice.J Exp Med.1999;190:915-922.

7. de Almeida Pdel V, Gregio AM, Machado MA, de Lima AA, Azevedo LR. Saliva composition and functions: a comprehensive review. J Contemp Dent Pract. 2008;9:72-80.

8. Brandtzaeg P. Do salivary antibodies reliably reflect both mucosal and systemic immunity? Ann N Y Acad Sci. 2007;1098:288-311.

9. Soo-Quee Koh D, Choon-Huat Koh G. The use of salivary biomarkers in occupational and environmental medicine. Occup Environ Med. 2007;64:202-210.

10. Nelson S, Kolls JK. Alcohol, host defence and society. Nat Rev Immunol. 2002;2:205-209.

11. Keedwell PA, Poon L, Papadopoulos AS, Marshall EJ, Checkley SA. Salivary cortisol measurements during a medically assisted alcohol withdrawal. Addict Biol. 2001;6:247-256.

12. Slomiany BL, Liau YH, Zalesna G, Slomiany A. Effect of ethanol on the in vitro sulfation of salivary mucin. Alcohol Clin Exp Res. 1988;12:774-779.

13. Ryder MI. The influence of smoking on host responses in periodontal infections. Periodontol 2000. 2007;43:267-277.

14. Waszkiewicz N,Zalewska-Szajda B,Zalewska A et al. Decrease in salivary lactoferrin output in chronically intoxicated alcohol-dependent patients. Folia Histochem Cytobiol. 2012;50: 248-254. 